\section{ON THE CAUSATION OF PUERPERAL CONVULSIONS.*}

By A. L. GALABIN, M.D., Assistant Obstetric Physician to Guy's Hospita .

IN any case of convulsions, under whatever circumstances it may arise, even the most careful study of the phenomena of the paroxysm affords little or no ground for inference as to the pathological process upon which they depend. Hence, even if the primary cause of any form of eclampsia be fully ascertained, there will still remain great difficulty in tracing the immediate antecedents of its outward manifestation. Nevertheless, it will be well to preface a brief consideration of puerperal convulsions by recalling the very few points which are known as to the pathology of convulsions in general. From the facts of physiology, it may be inferred that the immediate cause of the motor disturbance is a spasmodic and irregular discharge of nervous energy from the ganglia at the base of the brain, acting in conjunction with the medulla oblongata and spinal cord. Since in general those muscles are earliest and most strongly affected which are paralysed in hemiplegia due to disease of the corpus striatum, there is some reason for thinking that the corpus striatum may be the chief source of the paroxysmal discharge. In most cases, no organic changes are found in these parts on inspection; and, therefore, a convulsion is a phenomenon which points not to destroying lesion, but to instability of the nervous centres, resulting from some change in their structure too delicate to be recognised, which, therefore, falls within the definition of functional. Since unconsciousness generally accompanies the convulsion, we know that the cerebral hemispheres, as well as the motor centres, take part in the disturbance. For the determination of the question, Which of the centres takes the initiative in the explosion? some assistance may be derived from the experiments which are made for us by disease or by accident. When convulsions are the result of manifest organic changes in the brain, such change, in the great majority of cases, affects the grey matter of the convolutions on the surface. Thus, they are produced by tubercular or traumatic meningitis, by meningeal hæmorrhage, by tumour or abscess near the surface, and by syphilitic deposits, which almost invariably take place on the surface. Moreover, Dr. Wilks, in the Guy's Hospital Reports for 1866, has recorded a series of cases in which a disease, exactly similar in its manifestations to idiopathic epilepsy, was found to be associated with old standing disease of the membranes. On the other hand, disease or hremorrhage in the central ganglia produces not convulsion, but paralysis. It is reasonable, therefore, to conclude from analogy that, in all epileptiform attacks, even when not due to any visible lesion, the irritation commences at the surface of the convolutions ; and that in a convulsion, just as in voluntary motion, the energy of the motor centre is called out by a stimulus transmitted from the cerebral hemispheres.

It is more difficult to arrive at any certain conclusion with regard to the intimate nature of the process which takes place in the nerve-centres at the time of the convulsion. But the general principle is now accepted, that instability of nervous energy is a sign of enfeeblement of functional capacity. Further, we have the results of experiments on animals, showing that convulsions are produced by hæmorrhage, and the observation of Brown-Séquard and others, that, at the onset of an epileptiform fit, the nervous centres of an animal subjected to experiment become pale, instead of showing signs of congestion. This agrees with the fact that, at the commencement of a fit in the human subject, the face is pale, while the venous congestion which follows at a later stage is secondary to the muscular spasm and the interruption to respiration. It is, therefore, pretty certain that, at the outset of a fit. the state of the motor centres is that of anæmia rather than of congestion ; and there is, at least, considerable probability in the hypothesis of Dr. Russell Reynolds, that the immediate antecedent of the convulsion is a spasmodic contraction of the small arteries of the brain, as well as those of the face and other parts. It would not, however, necessarily follow from this that, as he supposes, the seat of primary derangement is in the medulla oblongata, for the vaso-motor centre may itself derive its irritation from the cerebral hemispheres.

With regard to the primary cause of puerperal eclampsia, since its association with albuminuria was first discovered by Dr. Lever in 1843 , the conclusion has been generally accepted that, in the great majority of cases, the most important element in its causation is uræmic poisoning of the blood. It is not difficult, on this view, to account for the fact that convulsions are much commoner, and form a much more predominant symptom, in this than in other forms of uræmia. For, in the first place, there is constantly at work during pregnancy, and especially

* Read at the Meeting of the East Surrey District of the South Eastern Branch. at the time of labour, a reflex nervous irritation. The importance of this is shown by the fact that, out of all the cases of puerperal convulsions, more than half make their first onset while labour is actually proceeding. And, in the second place, there is in pregnant women, as in children, a special liability to irritation from physical or emotional causes, and hence a proclivity to nervous diseases marked by convulsion. In his Lumleian lectures, delivered in 1873, Dr. Barnes has ably shown how this exalted state of reflex irritability is associated with the altered nutrition which is induced in the nervous centres by pregnancy, so as to provide a due supply of nerve-force for the work of parturition. The mode in which the effect of toxæmia may be combined with that of nerve-stimulus may be illustrated by the physiological action of those poisons, whose tendency is to produce convulsion. For, in the case, for instance, of strychnia, a dose of such an amount may be given to an animal that no convulsion is produced so long as it is kept perfectly undisturbed, but the slightest stimulus is sufficient to produce spasm.

With respect, however, to the association of albuminuria with puerperal eclampsia, a most important question was raised by Dr. Braxton Hicks, in a paper brought before the Obstetrical Society in 1867 . In this four cases were recorded, in which the urine examined before the onset of the convulsions, or immediately after the first fit, was free from albumen, while that passed a few hours later was highly albuminous. In one of the cases, in which a microscopic examination of the albuminous urine was made, it was found to contain waxy and epithelial casts and blood-corpuscles. Hence, a doubt is suggested by Dr. Hicks, whether the view which attributes eclampsia to uræmia may not be erroneous, and the true explanation be one of two alternatives, either that the nephritis and the convulsions are produced by the same cause, namely, some detrimental ingredient circulating in the blood, or else that the convulsion is primary, and that the albuminuria is due to the venous congestion produced by the spasm. The latter alternative had already been maintained by several German and French authors, and especially by Scanzoni. As regards the matters of fact of the absence of albuminuria at the earliest stage, statistics of a considerable number of cases would be desirable. But in the present paper I can only attempt to bring before you two or three cases of puerperal eclampsia and other forms of convulsions which have recentiy come under my notice, and to make them the text for a few remarks as to the connection of eclampsia with uremia and some other points in its pathology. I hope that the experience of some who are present to-day may afford further data bearing upon the questions at issue.

I would remark, first, that the albuminuria is not simply a passive transudation, the result of congestion, but it is the manifestation of a nephritis, generally of an acute character. This is apparent, even from the cases of Dr. Hicks, in which albumen was absent in the early stage, and it is still more evident when the more crucial test of post mortem evidence is applied. In the maternity charity of Guy's Hospital, we have very rarely the opportunity of making post mortem examinations; and, therefore, I will refer to the results published by Professor Braun of Vienna, whose experience is wider than that of most individuals, including fifteen fatal cases of puerperal eclampsia, in which an inspection was made. In seven of these, the kidneys were examined micro-, scopically, and evidence of nephritis found. In the remaining eight the microscope was not used, but the appearances to the naked eye were those of the first or second stage of nephritis. Again, out of four fatal cases recorded by Bourneville, parenchymatous nephritis was found in one ; and in the remaining three the appearances of acute tubal nephritis, namely, an opaque, swollen, yellowish white cortical substance and deeply congested pyramids.

In a considerable number of cases, we can be quite certain that the nephritis is antecedent in point of time to the eclampsia, for its result in the shape of œedema is manifest for a longer or shorter interval before the onset of the fits. In some, the albuminuria is detected, and the eclampsia is looked forward to with dread for months before it actually occurs. If, therefore, nephritis is the primary cause in some cases, it is $a$ priori unlikely that the relation of cause and effect should be inverted in the remainder. Again, if even a passive exudation of albumen were a secondary effect in puerperal convulsions, we should expect that albuminuria would also be produced by other epileptiform fits. It has been supposed by some that this is the case in epilepsy, but evidence appears to show the contrary. While taking temporary charge of the Cambridgeshire Lunatic Asylum, which contained many epileptics, some of whom had violent fits many times in the day, I took the opportunity of observing the effect of the fits on the urine and on the temperature. I did not find albumen in a single case ; and, in respect of temperature, I found in most cases a moderate elevation, perhaps not more than might be due to the effect of muscular action, and never passing beyond $100.5 \mathrm{deg}$. The absence of albumen was also noted in 
the following case, of which I will give brief notes, since, in its course and in its fatal result, it has a close analogy to some instances of puerperal convulsions.

Jane V., aged I I, was suddenly attacked by an epileptiform fit at 6 P.M. on November 27 th, 1873 . She had never had fits previously, except that, when an infant, she had a severe attack of convulsions during dentition. As she remained insensible after the fit, she was at once brought to Guy's Hospital. On her admission, she was completely comatose ; respirations 48 , rattling; pulse 136 ; temperature, I00. 3 deg. She remained in this condition without any recurrence of convulsions until about midnight, when another fit occurred, and from that time they became gradually more frequent. At I A.M., the temperature was I03.7 deg.; pulse I56; respirations 64 . At 9.30 A.M., the venous congestion, which had all along been marked after each convulsion, began to remain permanently through the intervals. Temperature, I05. 7 deg.; pulse $\mathbf{I} 68$; respirations 56 . The urine was withdrawn by a catheter at this time and examined. Its specific gravity was 1024, and it was loaded with urates, but contained no albumen nor sugar. At 12.10, the temperature was 108.2 ; at 12.45, 109.1 deg., and at this tinie the convulsive movements were becoming almost continuous, and the venous congestion was extreme. A vein was opened in the arm, but only four ounces of blood could be procured. The effect, however, was that the convulsions ceased, and the temperature in axilla fell to $103.8 \mathrm{deg}$. She died, however, half an hour later. Three quarters of an hour after death, the temperature in the vagina was Iog deg. Post mortem, nothing abnormal could be detected in the brain, kidneys, or any other organ.

This case seems to be one in which it might be expected that albuminuria would have occurred, if convulsions were capable of producing such a result. The most remarkable feature, however, is the occurrence of hyperpyrexia; and in this respect, as well as in the duration of its course up to the fatal issue, it has a close resemblance to the following case of puerperal eclampsia.

Eliza H., a primipara, aged 20, a patient in the Guy's Hospital Charity, was attended by an extern, and was delivered about $3 \mathrm{~A} . \mathrm{M}$. on November 23rd, I874. About ten minutes after the birth of the child, she had a convulsion, but soon recained consciousness. At Io A.M., she had had five more fits, and had become unable to answer questions, but her pulse was only 65 , and her state did not excite the extern's alarm. The its afterwards became more frequent, and the patient's friends, not finding the extern at home, instead of sending to the hospital, called in another medical man. In this way, it happened that she was not seen by the obstetric resident until 7 P.M. At that time, he found her completely comatose ; temperature in axilla, Io7 deg. ; respirations 65 ; pulse 130 . The urine, obtained by a catheter, was dark, contained a large quantity of albumen, and many granular and hyaline casts; specific gravity IO2 I. The surface of the body was then sponged with water at 95 deg. Fahr.; and in this way the temperature in the axilla was reduced to IO4 deg.; but the temperature in the vagina was found to be IOS.8 deg., almost exactly the same point as that reached in the case last recorded. In the meantime I had been summoned, but on my arrival I found the patient had just died. No necropsy could be procured.

I have not been able to find any reference to the temperature in puerperal eclampsia in the English-text-books on the subject; but in several other cases which ended in recovery I have found a considerable but not an extreme elevation. I will give very brief notes of one of them.

On January $13^{\text {th }}$, I 873 , I was called to a primipara, aged 23 , who had had a convulsion an hour and a half after delivery. The urine was withdrawn by a catheter, and was found to be loaded with albumen, and to contain numerous hyaline and epithelial casts. There was no œdema at that time nor previously. Temperature ror. $8 \mathrm{deg}$.; pulse IO4. The patient was quite conscious, and was treated by a thirty-grain dose of chloral, two other similar doses being given at intervals of four hours. She had one more fit about two hours later, after which the temperature was noted to be I02.6 deg.; pulse IIo. The con vulsions did not recur after this, and the temperature gradually returned to its normal value. At the end of five days, the urine was quite free f́rom albumen.

Some remarkable observations on temperature in puerperal eclampsia have lately been published by Bourneville, which tend to show that there is always considerable elevation, and that the hyperpyrexia which occurred in the instance which I have recorded is not exceptional, but is the rule in fatal cases. Bourneville reports five cases in which re covery took place, and three which were fatal. In the former, the temperatures reached were respectively 104.4, I02.6, I04.4, IO3. I, 102.4 deg. In the fatal cases the temperatures continued increasing up to the time of death, while the patient remained comatose, and the maxima reached were 109.6, 106, 108.6 deg., so that in one of these a somewhat higher point was reached than in the two cases of hyperpyrexia associated with convulsions which I have recorded.

It appears to me that these facts lead to a practical conclusion, the importance of which has not yet been recognised. I think that we may derive the lesson, that in the treatment of puerperal eclampsia the thermometer should be constantly used, and that its indications will afford an important guide to the gravity of the peril. Moreover, it may be concluded that, if the temperature should rise as high as I05 deg., recourse should be had to baths or to sponging the surface of the body, in addition to the use of chloroform. In the somewhat analogous case of hyperpyrexia in acute rheumatism, experience has already shown that by cooling the body some patients may be saved who would otherwise inevitably have died.

A remarkable contrast in respect of temperature between puerperal eclampsia and other forms of uræmia appears from the observations of Bourneville and others. In uræmia, with coma, there is generally a depression of temperature, which in some cases is very considerable. Dr. Wr. Roberts records a case of uræmia from granular kidney, in which the temperature ranged between 94.6 and $96.6 \mathrm{deg}$, , and another of suppression of urine from a calculus impacted in the ureter, in which it fell to $97 \mathrm{deg}$. Bourneville reports three cases of uræmia with coma, in which the temperatures reached were $86.2,86.6,91.6 \mathrm{deg}$. These temperatures were taken in the rectum, and all the cases were fatal. In two cases recorded by Bourneville, in which uræmia was accompanied by convulsions, the minima of temperature were $98.2,95.8 \mathrm{deg}$. The depression was, therefore, not so great as in uræmia without eclampsia. My own observations, so far as they have gone, indicate a similar conclusion, although I have never found so considerable a diminution of temperature. In two cases of uræmia with coma, ending in recovery, I have found a temperature in the axilla ranging from 96 to $97 \mathrm{deg}$; ; and in two cases of uræmia with occasional convulsions, in which recovery also took place, a temperature of from 97 to $98.5 \mathrm{deg}$. The explanation may probably be that convulsions have a tendency to raise the temperature, and uræmia to lower it, and thus the occasional eclamptic attacks in ordinary cases of uræmia only serve to modify the depression. In puerperal cases, however, on account of the increased susceptibility of the nervous system to irritation, a very slight amount of uræmia, especially when it is not the result of chronic disease, but arises suddenly, and so finds the system unprepared, is sufficient to produce an extreme degree of the convulsive state. Thus, the latter condition predominates, and the temperature is elevated. In cases of ordinary epilepsy, the observations of Bourneville agree with those made by myself, to which I have before referred, in showing that a decided, but not considerable, elevation of temperature generally occurs.

I will add a short account of another case of eclampsia, fortunate in its issue, which, like the others, shows an elevaticn of temperature, and which also affords evidence as to the state of the vascular system at the time of the convulsions.

Emma C., aged 42, a pluripara, having previously had seven children, had never had convulsions in any former labours. On October 3 rd, 1874, when about eight months pregnant, after slight premonitory symptoms of headache and malaise, she was found in a fit of convulsions, which lasted about five minutes, and left her in a semicomatose state. She had noticed slight œedema of the ankles a fortnight previously. During the day, she had four other fits; and at 4 P.M. was brought to Guy's Hospital. On admission, her face was puffy, and complexion sallow and anæmic, resembling that seen in Bright's disease. She was quite unconscious, the eyes staring and vacant, the pupils dilated and almost insensible to light ; the extremities were cold; pulse 96 ; temperature in axilla IOI.2 deg. A fit occurred soon after her admission, and another half an hour later. The urine was drawn off by a catheter, and found to be highly albuminous; specific gravity roro. No casts were found. In the absence of Dr. Braxton Hicks, I was summoned to see the case. I directed a castor-oil injection to be given, and chloroform to be administered. This was at first given fully, and afterwards was occasionally resumed whenever she became very restless. At this time, I took a sphygmographic tracing of the pulse. The instrument was with difficulty applied before chloroform was commenced; but, from the struggles of the patient, it was impossible to obtain a satisfactory tracing. From watching the movements of the lever, it appeared that the amplitude of the pulse was small, and that it required a pressure of six ounces, the usual pressure required for a healthy pulse being about three ounces. When chloroform had been partially given, a tracing was obtained at a pressure of three ounces, showing a broad rounded systolic summit, which denotes a prolonged systole of the heart, generally due to obstructed outflow from the arteries. When chloroform had been fully given, the pulse took a pressure of only two ounces, and the trace was more ample and more dicrotic. Hence, the 
chloroform produced a rapid lowering of the excessive arterial pressure.

At 8 P.M., the patient was still insensible, although much quieter; temperature 102.3. The os uteri was high up, and just admitted one finger into the cervical cavity. I then decided to commence the induction of premature labour, which I did by rupturing the membranes with a stylet.

At I I P.M., the fits had not recurred; she was somewhat more sensible, and occasionally answered questions when addressed. The labour had not made any advance. Temperature 99.2 ; pulse 108 . At 9 A.M. on the 4 th, she had no more convulsions, and was much more sensible. The urine was very slightly albuminous; specific gravity ror 5 . At 5 P.M., the os was high up, and not more dilated; the vertex was not presenting, but the presentation could not be fairly made out. No pains had occurred. At 9 P.M., the obstetric resident was summoned, and found her in a wild, excited, semiunconscious state, with both feet of the child protruding from the vulva, the cord not pulsating. Chloroform was given, and in a few minutes the head was extracted. No more convulsions occurred ; and on the 8th, the urine contained no albumen nor casts; specific gravity I020. She recovered rapidly.

It is to be noticed in the course of this case that a considerable improvement followed the use of chloroform; but a still more decided benefit from the diminution of nerve-irritation obtained by the reduction in size of the uterus at the escape of the liquor amnii, although labour did not come on until twenty-four hours later. The temperature had continued to rise after the use of chloroform, but fell three degrees in three hours after the rupture of the membranes. The chief reason, however, for which I bring it before you, is because it shows that high arterial tension existed at the time of the convulsions, and before chloroform was administered. Now, it has been shown by the use of the sphygmograph, that the same state of the vascular system exists in chronic, and to some extent even in acute Bright's disease. It is attributed to a resistance to the circulation of the blood, either directly the consequence of its altered quality, or due to a spasmodic contraction of the small arteries, set up by its irritating effect. Now, in several cases of uræmia, arising in the course of chronic or acute Bright's disease, I have found that while the temperature was diminished, the pulse became rapid; but, at the same time, the sphygmograph showed that it remained non-dicrotic, and bore a pressure sometimes greater than before, and considerably greater than normal. As a rule, when the pulse becomes rapid, it becomes dicrotic and compressible. 'Thus it seemed, that during the uramic state, there was a still further enhancement of that elevated arterial tension which is usual in Bright's disease, and the condition in the case of puerperal eclampsia just related was somewhat similar. It is possible, therefore, that in this circumstance may be found some explanation of the beneficial action of chloroform, which in that case produced, as it usually does, arterial relaxation. It probably acts by soothing the irritability of those nervous centres which excite the spasmodic action of the vaso-motor nerves, and either through their instrumentality, or by other means, produce convulsions and coma. A diminution of arterial tension is also one of the results of bleeding, and perhaps it is in this way that venesection has proved beneficial, as well as by causing that diminution of temperature which is known to be at least a temporary effect of the abstraction of blood. The dictates of fashion have now declared decisively against bleeding in puerperal convulsions, and no doubt it is true that, in chloroform, there has been found a less injurious means of relaxing arterial spasm, and probably in the application of cold a better mode of reducing the temperature. Nevertheless, it seems probable, that in those cases in which venous congestion has become continuous, and the temperature is rising to a high point, venesection may still be found useful to relieve the strain upon heart and lungs; while, at the same time, it tends to lower temperature and decrease arterial tension. If time allow, however, a considerable part of the same effect, including that of the removal of some of the morbid matter in the blood, may be obtained by free purgation.

With the increase of arterial tension in puerperal eclampsia may be associated the fact that such an increase of tension in minor degree is a normal, or, at any rate, a common condition in pregnancy. This is indicated sphygmographically by an increase in the tidal wave in the pulse, and in the pressure required for its development. It seems to be attributable to the presence in the blood of some irritating material, so that its behaviour is like that of blood which kidneys, deficient in excretory power, have failed to purify. I think that this condition throws light upon the causation of nephritis in pregnancy. It has often been described as being due to mechanical pressure; but, although this no doubt takes some part in producing the result, yet there are several reasons for considering that it is insufficient in itself to account for the whole. For the nephritis, and even the convulsions, are sometimes found to occur as early as the third or fourth month of pregnancy, when pressure could produce little effect; and experience shows also that in cases of ovarian or other tumours, which may produce even greater pressure than pregnancy,albuminuria is very rare, and nephritis still rarer. Again, heart-disease may produce a still greater venous congestion, and albuminuria may result ; but the kidneys do not show the signs of inflammation which are found in fatal cases of puerperal eclampsia. It would seem, then, that the nephritis of pregnancy is due in part to the presence in the blood of some deleterious material, which also causes an elevation of arterial tension. It has been shown by Mr. Mahomed, in a paper read last year before the Royal Medical and Chirurgical Society, that a similar elevation of tension occurs in scarlatina, and forms a stage antecedent to that of albuminuria.

It is extremely unlikely, however, that in puerperal cases this in-jurious material in the blood should be developed so suddenly as to produce directly and independently both eclampsia and a fully developed nephritis within the space of a few hours. And the facts brought forward by Dr. Hicks, showing that the appearance of albumen in the urine in some cases does not precede the eclampsia, or even follow it after an interval of an hour or two, do not, when rightly considered, prove that nephritis is not the cause of the convulsions. For, although albuminuria is the chief available sign of kidney-disease, it is not necessarily, either according to logic or experience, its first effect. Thus we know that chronic degeneration of the kidney may exist for some time, and produce decided effects upon the system, before any albuminuria occurs; and, again, cases are known of scarlatinal dropsy, in which the dropsy precedes by some hours, or by even a day, the appearance of albumen in the urine. As, therefore, in that case, the failure in the excretion of water precedes the albuminuria, so, in the case of puerperal nephritis, may the loss of power to excrete solid matters.

I would draw, therefore, the final conclusion that there is much ground for the conjecture that the primary cause, both of puerperal eclampsia and of albuminuria, is the presence of some injurious matter circulating in the blood; but that there is strong evidence to lead us to believe that it is not sufficient by itself to produce the convulsions, until nephritis has actually commenced, although in a few cases it may not yet have reached the stage of albuminuria. It is to be remembered, at the same time, that now and then, although very rarely, cases of puerperal convulsions occur in which no albumen can be found in the urine either before or after the fits. The pathology of these would seem to be entirely different, and to be more nearly allied to that of ordinary: epilepsy.

\section{CASE OF CONGENITAL SYPHILIS, WITH ASCITES, IN A CHILD THREE MONTHS OLD : DEATH : POST MORTEM EXAMINATION.}

By IV. B. CIIEADLE, M.D., F.R.C.P., Senior Assistant-Physician to and Lecturer on Pathology at St. Mary's Hospital; Senior Assistant-Physician to the Hospital for Sick Children.

O. A. II., a boy aged three months, was brought to me at the Hospital for Sick Children in Great Ormond Street on April 24th, 1874. The child's face showed the yellow "bistre" tint very conspicuously; it had snuffles and much acrid discharge from the nose, which was excoriated. The buttocks and genitals were covered with a dark-red dry erythematous eruption. The legs and feet were highly œedematous, and the abdomen distended to a great size with fluid ; the navel being very protuberant. The liver was large. The child was much emaciated, but this was so masked by the odema that it had not been specially noticed by the mother. It was ascertained that the latter, a most respectable healthy-looking woman, had had two miscarriages, and four years ago had given birth to a stillborn child, which the doctor stated must have been dead for a month.

The child was said to take its food well, and supposed to be in fair health, but for the dropsy and the "thrush", or eruption on the genitals and buttocks. It was ordered a grain of grey powder every four hours, and mercurial ointment was directed to be rubbed in night and morning. When next seen, at the expiration of a week, it was reported to be better in every way. The odema had decreased in a remarkable manner, and the ascites was also less. The extremely emaciated condition of the child, with the skin hanging in loose folds on its arms and legs, was now rendered very manifest by the subsidence of the dropsy. The same treatment was continued with apparent advantage, the dropsy regularly diminishing until the limbs were free from odema. There was no purging, nor were the stools green, but the bowels were regular and the motions natural. On May 8th, however, a fortnight after its admission, it was seized with convulsions, and died the same evening. 